\title{
Finite deformations of Ogden's materials under impact loading
}

\author{
Z.-Q. Feng ${ }^{\mathrm{a}, *}$, F. Peyraut ${ }^{\mathrm{b}}$, Q.-C. He ${ }^{\mathrm{c}}$ \\ a Laboratoire de Mécanique d'Évry, Université d'Évry-Val d'Essonne, 40 rue du Pelvoux, 91020 Évry, France \\ ${ }^{\mathrm{b}}$ LERMPS, Université de Technologie de Belfort-Montbéliard, Sévenans, 90010 Belfort, France \\ ${ }^{\mathrm{c}}$ Laboratoire de Mécanique, Université de Marne-la-Vallée, 19 rue A. Nobel, 77420 Champs sur Marne, France
}

\begin{abstract}
The present paper is devoted to the modeling of finite deformations of a hyperelastic body described by Ogden's model under contact/impact conditions. Frictional contact problems are solved by means of the bi-potential method. The first order algorithm is applied to integrate the equation of motion. The total Lagrangian formulation is adopted to describe the geometrically non-linear behavior. For the finite element implementation, the explicit expression of the tangent operator is derived including the case of repeated eigenvalues. A numerical example is given to illustrate efficiency and accuracy of the method.
\end{abstract}

Keywords: Hyperelasticity; Contact/impact; Ogden's model; Large deformation

\section{Introduction}

Problems involving unilateral contact and friction are among the most difficult ones in mechanics and at the same time of crucial importance in many engineering branches. A large number of algorithms for the modelling of contact problems by the finite element method have been presented in the literature. See, for example, the monographs by Kikuchi and Oden [1], Zhong [2] and Wriggers [3], and the references therein. The bipotential method proposed by De Saxcé and Feng [4,5] turns out to be particularly efficient for the treatment of quasi-static frictional contact problems [6-9]. The present work extends the bi-potential method to dynamic contact problems by using a first-order time integration algorithm and considering a class of hyperelastic materials which can be described by the wellknown Ogden's constitutive law [10].

Dynamic contact or impact falls within the framework of non-linear dynamics. The computational treatment of a nonlinear dynamic problem must comply with the basic requirement that: (i) the linear and angular momenta be conserved; (ii) the energy be conserved when the corresponding phenomenon

\footnotetext{
* Corresponding author. Tel.: +33169477501; fax: +33169477599.

E-mail address: feng@iup.univ-evry.fr (Z.-Q. Feng).
}

is non-dissipative; and (iii) the energy dissipation be exactly equal to the physical one when the corresponding phenomenon is dissipative. The problem of satisfying these three requirements in the case of dynamic contact has been addressed and dealt with in a few recent computational mechanics works [11-14].

In the present work, this problem is solved by using the bipotential method together with the first-order time integration algorithm which was applied by Jean [15] to simulate nonsmooth dynamic contact of granular materials. In fact, the firstorder time integration is both simple and suitable for discontinuous phenomena such as impact [16].

In non-linear elasticity, there exist many models to describe the hyperelastic behavior of foam- or rubber-like materials [10,17-22]. The Ogden's model plays an important role in modelling such materials and has been implemented in most commercial finite element codes. However, it seems that the problem of finite deformations of Ogden's materials under impact loading has not been investigated from a computational point of view. Consequently, the main purpose of the present paper is to study and solve this problem by means of the bi-potential method together with the first-order time integration algorithm.

This paper is structured as follows. In Section 2, the problem of frictional dynamic contact of Ogden's bodies is 
formulated. Next, the bi-potential method is recapitulated in Section 3. The stress-strain tangent operator associated to the hyperelastic Ogden's law is calculated in Section 4. The finite element formulation of the frictional dynamic contact problem and the first-order time integration algorithm are given in Section 5. A numerical example concerning the impact of a hyperelastic cylinder into a funnel is provided in Section 6 to show the energy conservation in the absence of friction and the energy dissipation in the presence of friction. In Section 7, a few concluding remarks are drawn.

\section{Problem setting}

In this section, basic definitions and notations used are briefly described. Details can be found in [3].

\subsection{Kinematics}

Consider a deformable body $\mathscr{B}$ undergoing large strains (Fig. 1). The body $\mathscr{B}$ occupies in its initial configuration an open, simply connected and bounded domain $\Omega_{0} \subset \mathbb{R}^{3}$. The closure $\bar{\Omega}_{0}$ of $\Omega_{0}$ represents the reference configuration of $\mathscr{B}$ in the total Lagrangian formulation, adopted here. A material particle $\mathscr{M}$ of $\mathscr{B}$ is identified by its reference position vector $\mathbf{X} \in \bar{\Omega}_{0}$. At each time $t \in \mathbf{I}_{t}$, where $\mathbf{I}_{t}:=[0, T]$ denotes the time interval of a loading process, the boundary $\Gamma$ of $\bar{\Omega}_{0}$ can, in general, be split into three parts: $\Gamma_{u}$ with prescribed displacements $\overline{\mathbf{u}}, \Gamma_{t}$ with prescribed tractions $\overline{\mathbf{t}}$, and the potential contact surface $\Gamma_{c}$, where the body $\mathscr{B}$ may come into contact with the surface $\Gamma^{\prime}$ of a rigid body $\mathscr{B}^{\prime}$ :

$\Gamma=\Gamma_{u} \cup \Gamma_{t} \cup \Gamma_{c}$.

With no loss of generality, the rigid body is assumed to be motionless.

The successive deformed configurations $\Omega(t)$ of $\mathscr{B}$ with $t \in$ $\mathbf{I}_{t}$ are defined by a one-parameter differentiable and invertible mapping $\varphi$ :

$\varphi(\mathbf{X}, t): \bar{\Omega} \times \mathbf{I}_{t} \rightarrow \mathbb{R}^{3}$.

The boundary $\Gamma(t)$ of $\Omega(t)$ is assumed to be sufficiently smooth so that an outward unit normal vector, denoted by $\mathbf{m}$, can be defined almost everywhere on $\Gamma$. The position of a particle of the body in the current configuration $\Omega(t)$ is defined by its current position vector $\mathbf{x}=\varphi(\mathbf{X}, t)$. The displacement of a material point corresponds to the difference between its current and initial positions

$\mathbf{u}=\varphi(\mathbf{X}, t)-\mathbf{X}$.

The velocity and acceleration of a material point in the Lagrangian description are given as

$\dot{\mathbf{u}}(\mathbf{X}, t)=\frac{\partial \varphi}{\partial t}(\mathbf{X}, t), \quad \ddot{\mathbf{u}}(\mathbf{X}, t)=\frac{\partial^{2} \varphi}{\partial t^{2}}(\mathbf{X}, t)$.

The transformation gradient $\mathbf{F}$ is defined by

$\mathbf{F}(\mathbf{X}, t)=\frac{\partial \mathbf{x}(\mathbf{X}, t)}{\partial \mathbf{X}}=\mathbf{I}+\frac{\partial \mathbf{u}(\mathbf{X}, t)}{\partial \mathbf{X}}$,



Fig. 1. Contact kinematics.

where $\mathbf{I}$ is the unity tensor, and is required to be such that $J=\operatorname{det}(\mathbf{F})>0$. The constraint of incompressibility (isochoric deformation) is characterized by

$J=\operatorname{det}(\mathbf{F})=1$.

The strain measure adopted here is the Green-Lagrangian strain tensor $\mathbf{E}$ :

$\mathbf{E}=\frac{1}{2}(\mathbf{C}-\mathbf{I})$,

where $\mathbf{C}=\mathbf{F}^{\mathrm{T}} \mathbf{F}$ is the right Cauchy-Green tensor.

\subsection{Ogden's hyperelastic law}

The deformable body under consideration is assumed to be rubber-like and more precisely characterized by Ogden's hyperelastic law. Let $W$ be the strain energy density which is a scalar function of either the strain tensor $\mathbf{E}$ or $\mathbf{C}$. Thus, the second Piola-Kirchhoff stress tensor $\mathbf{S}$ is given by

$\mathbf{S}=\frac{\partial W}{\partial \mathbf{E}}=2 \frac{\partial W}{\partial \mathbf{C}}$

In the case of isotropic hyperelasticity [18], Eq. (7) can be written as

$\mathbf{S}=2\left[I_{3} \frac{\partial W}{\partial I_{3}} \mathbf{C}^{-1}+\left(\frac{\partial W}{\partial I_{1}}+I_{1} \frac{\partial W}{\partial I_{2}}\right) \mathbf{I}-\frac{\partial W}{\partial I_{2}} \mathbf{C}\right]$,

where $I_{i}(i=1,2,3)$ denote the invariants of the right Cauchy-Green deformation tensor $\mathbf{C}$ :

$I_{1}=\operatorname{tr}(\mathbf{C}), \quad I_{2}=\left(I_{1}^{2}-\mathbf{C}: \mathbf{C}\right) / 2, \quad I_{3}=\operatorname{det}(\mathbf{C})=J^{2}$.

In terms of the eigenvalues (or principal stretches) $\lambda_{i}(i=1,2,3)$ of the right Cauchy-Green tensor $\mathbf{C}$, we have

$I_{1}=\lambda_{1}+\lambda_{2}+\lambda_{3}, \quad I_{2}=\lambda_{1} \lambda_{2}+\lambda_{2} \lambda_{3}+\lambda_{1} \lambda_{3}$,

$I_{3}=\lambda_{1} \lambda_{2} \lambda_{3}$. 
In this paper, Ogden's constitutive law is adopted [10]. Correspondingly, $W$ is expressed by

$$
\begin{aligned}
W\left(\lambda_{1}, \lambda_{2}, \lambda_{3}\right)= & \sum_{i=1}^{N} \frac{\mu_{i}}{\alpha_{i}}\left(\lambda_{1}^{\alpha_{i} / 2}+\lambda_{2}^{\alpha_{i} / 2}+\lambda_{3}^{\alpha_{i} / 2}-3\right) \\
& +\sum_{i=1}^{N} \frac{\mu_{i}}{\alpha_{i} \beta_{i}}\left(J^{-\alpha_{i} \beta_{i}}-1\right),
\end{aligned}
$$

where $N, \mu_{i}, \alpha_{i}$ and $\beta_{i}$ are material parameters. The initial shear modulus, $G$, and the initial bulk modulus, $K$, are given as

$G=\frac{1}{2} \sum_{i=1}^{N} \mu_{i} \alpha_{i}, \quad K=\sum_{i=1}^{N} \mu_{i} \alpha_{i}\left(\frac{1}{3}+\beta_{i}\right)$.

It is noted that, for $N=1, \mu_{1}=-G, \alpha_{1}=-2$ and $\beta_{1}=0.5$, the well-known Blatz-Ko strain-energy density is recovered [17]

$W=\frac{G}{2}\left(\lambda_{1}^{-1}+\lambda_{2}^{-1}+\lambda_{3}^{-1}+2 J-5\right)$

or equivalently

$W=\frac{G}{2}\left(\frac{I_{2}}{I_{3}}+2 J-5\right)$.

Some other hyperelastic models are discussed by Horgan and Saccomandi [22].

By deriving the energy density (11) with respect to the strain tensor, we obtain

$$
\begin{aligned}
\mathbf{S}= & \sum_{i=1}^{N} \mu_{i}\left(\lambda_{1}^{\left(\alpha_{i} / 2\right)-1} \frac{\partial \lambda_{1}}{\partial \mathbf{C}}+\lambda_{2}^{\left(\alpha_{i} / 2\right)-1} \frac{\partial \lambda_{2}}{\partial \mathbf{C}}+\lambda_{3}^{\left(\alpha_{i} / 2\right)-1} \frac{\partial \lambda_{3}}{\partial \mathbf{C}}\right) \\
& -\sum_{i=1}^{N} \mu_{i} J^{-\alpha_{i} \beta_{i}} \mathbf{C}^{-1} .
\end{aligned}
$$

Using the Cayley-Hamilton theorem, we can write (15) in the equivalent form

$$
\begin{aligned}
\mathbf{S}= & \sum_{i=1}^{N} \mu_{i}\left(\lambda_{1}^{\left(\alpha_{i} / 2\right)-1} \frac{\partial \lambda_{1}}{\partial \mathbf{C}}+\lambda_{2}^{\left(\alpha_{i} / 2\right)-1} \frac{\partial \lambda_{2}}{\partial \mathbf{C}}+\lambda_{3}^{\left(\alpha_{i} / 2\right)-1} \frac{\partial \lambda_{3}}{\partial \mathbf{C}}\right) \\
& -\sum_{i=1}^{N} \mu_{i} J^{-\alpha_{i} \beta_{i}-2}\left(\mathbf{C}^{2}-I_{1} \mathbf{C}+I_{2} \mathbf{I}\right) .
\end{aligned}
$$

The first Piola-Kirchhoff stress tensor $\mathbf{P}$ and Cauchy stress (or true stress) tensor $\boldsymbol{\sigma}$ are related to the second Piola-Kirchhoff one $\mathbf{S}$ as follows

$\mathbf{P}=\mathbf{F S}, \quad \boldsymbol{\sigma}=\frac{1}{J} \mathbf{F S F}^{\mathrm{T}}$.

\subsection{Frictional unilateral contact conditions}

Consider a point $\mathbf{x}=\varphi(\mathbf{X}, t)$ with $\mathbf{X} \in \Gamma_{c}$ at an instant $t \in$ $\mathbf{I}_{t}$. The orthogonal projection of $\mathbf{x}$ on the rigid body surface $\Gamma^{\prime}$ is defined by $\mathbf{x}^{\prime}$. The contact distance vector (or gap vector function) is defined by

$\mathbf{g}=\mathbf{x}-\mathbf{x}^{\prime}=h \mathbf{n}$, where $\mathbf{n}$ is the outward unit normal vector of $\Gamma^{\prime}$ at $\mathbf{x}^{\prime}$ and $h$ is the oriented contact distance, specified by $h=\left\|\mathbf{x}-\mathbf{x}^{\prime}\right\|$. The plane $\mathscr{S}$ tangent to $\Gamma^{\prime}$ at $\mathbf{x}^{\prime}$ is defined by

$\mathscr{S}=\left\{\mathbf{y} \in \mathbb{R}^{3} \mid\left(\mathbf{y}-\mathbf{x}^{\prime}\right) \cdot \mathbf{n}=0\right\}$.

Let $\mathbf{r}$ be the contact stress vector exerted by $\Gamma^{\prime}$ on $\mathscr{B}$ at $\mathbf{x}$. Next, the displacement vector $\mathbf{u}$, the velocity vector $\dot{\mathbf{u}}$ and the contact stress vector $\mathbf{r}$ at $\mathbf{x}$ can be uniquely decomposed into a normal part and a tangential part as follows:

$\mathbf{u}=\mathbf{u}_{t}+u_{n} \mathbf{n}, \quad u_{n}=\mathbf{u} \cdot \mathbf{n}, \quad \mathbf{u}_{t}=(\mathbf{I}-\mathbf{n} \otimes \mathbf{n}) \mathbf{u}$,

$\dot{\mathbf{u}}=\dot{\mathbf{u}}_{t}+\dot{u}_{n} \mathbf{n}, \quad \dot{u}_{n}=\dot{\mathbf{u}} \cdot \mathbf{n}, \quad \dot{\mathbf{u}}_{t}=(\mathbf{I}-\mathbf{n} \otimes \mathbf{n}) \dot{\mathbf{u}}$,

$\mathbf{r}=\mathbf{r}_{t}+r_{n} \mathbf{n}, \quad r_{n}=\mathbf{r} \cdot \mathbf{n}, \quad \mathbf{r}_{t}=(\mathbf{I}-\mathbf{n} \otimes \mathbf{n}) \mathbf{r}$.

The unilateral contact law is characterized by a geometric condition of non-penetration, a static condition of no-adhesion and a mechanical complementary condition. These three conditions, known as the Signorini conditions, can be formulated as

$h \geqslant 0, \quad r_{n} \geqslant 0, \quad h r_{n}=0$.

In general, at any time $t \in \mathbf{I}_{t}$, the potential contact surfaces $\Gamma_{c}$ can be split into two disjoint parts: ${ }^{+} \Gamma_{c}$ where the body is in contact with $\Gamma^{\prime}$ and ${ }^{-} \Gamma_{c}$ where the body is separated from $\Gamma^{\prime}$. In the case of dynamic contact, the Signorini conditions can be formulated, on ${ }^{+} \Gamma_{c}$, via the relative velocity

$\dot{u}_{n} \geqslant 0, \quad r_{n} \geqslant 0, \quad \dot{u}_{n} r_{n}=0$ on ${ }^{+} \Gamma_{c}$.

The bodies are separating when $\dot{u}_{n}>0$ and remain in contact for $\dot{u}_{n}=0$.

Formulation (24) of the Signorini conditions can be combined with the sliding rule to derive the complete frictional contact law for the contacting part ${ }^{+} \Gamma_{c}$. This complete law specifies possible velocities of bodies that satisfy the unilateral contact conditions and the sliding rule. In this work, the classical isotropic Coulomb friction rule is used. The set of admissible forces, denoted by $K_{\mu}$, is defined by

$K_{\mu}=\left\{\mathbf{r} \in \mathbb{R}^{3}\right.$ such that $\left.\left\|\mathbf{r}_{t}\right\|-\mu r_{n} \leqslant 0\right\}$,

which is Coulomb's convex cone. On the contacting surface ${ }^{+} \Gamma_{c}$, the sliding rule can be combined with the rate form of the Signorini conditions to obtain the frictional contact law that specifies possible scenarios on the contact area (stick, slip, separation). Two overlapped "if...then...else" statements can be used to write it in a compact form:

$$
\begin{array}{lll}
\text { if } & r_{n}=0 \text { then } \dot{u}_{n} \geqslant 0 & \text { ! separating } \\
\text { elseif } & \begin{array}{l}
\mathbf{r} \in \text { int } K_{\mu} \text { then } \dot{u}_{n}=0 \\
\text { and }-\dot{\mathbf{u}}_{t}=\mathbf{0}
\end{array} & \text { ! sticking } \\
\text { else } & \begin{array}{l}
\left(\mathbf{r} \in \text { bd } K_{\mu} \text { and } r_{n}>0\right) \\
\end{array} & \left\{\begin{array}{l}
\dot{u}_{n}=0 \text { and } \exists \dot{\lambda}>0 \text { such that } \\
\end{array}\right. \\
& \left.-\dot{\mathbf{u}}_{t}=\dot{\lambda} \frac{\mathbf{r}_{t}}{\left\|\mathbf{r}_{t}\right\|}\right\} & \text { ! sliding }
\end{array}
$$

endif

where "int $K_{\mu}$ " and "bd $K_{\mu}$ " denote the interior and the boundary of $K_{\mu}$, respectively. The multivalued character of the law 
lies in the first and the second part of the statement. If $r_{n}$ is null then $\dot{\mathbf{u}}$ must be such that its normal component $\dot{u}_{n}$ should be positive. In other words, one single element of $\mathbb{R}^{3}(\mathbf{r}=\mathbf{0})$ is associated with an infinite number of velocity vectors $\dot{\mathbf{u}} \in \mathbb{R}^{3}$. The same arguments remain valid for the second part of the statement.

It is noted that the minus sign before $\dot{\mathbf{u}}_{t}$ means that the frictional force is opposite to the sliding velocity in the isotropic friction case. The complete form of the frictional contact law involves three possible states, which are separating, contact with sticking, and contact with sliding. Only the last state produces energy dissipation.

\section{The bi-potential method}

De Saxcé and Feng [5] have shown that the contact law (26) is equivalent to the following differential inclusion:

$$
\left(-\dot{\mathbf{u}}_{t}-\left(\dot{u}_{n}+\mu\left\|-\dot{\mathbf{u}}_{t}\right\|\right) \mathbf{n}\right) \in \partial \bigcup_{K_{\mu}} \mathbf{r}
$$

where $\bigcup_{K_{\mu}} \mathbf{r}$ denotes the so-called indicator function of the closed convex set $K_{\mu}$ :

$\bigcup_{K_{\mu}}(\mathbf{r})= \begin{cases}0 & \text { if } \mathbf{r} \in K_{\mu} \\ +\infty & \text { otherwise }\end{cases}$

The following contact bi-potential is obtained:

$b_{c}(-\dot{\mathbf{u}}, \mathbf{r})=\bigcup_{\mathbb{R}_{-}}\left(-\dot{u}_{n}\right)+\bigcup_{K_{\mu}}(\mathbf{r})+\mu r_{n}\left\|-\dot{\mathbf{u}}_{t}\right\|$,

where $\left.\left.\mathbb{R}_{-}=\right]-\infty, 0\right]$ is the set of the negative real numbers. In order to avoid non-differentiable potentials that occur in nonlinear mechanics, such as in contact problems, it is convenient to use the augmented Lagrangian method [4-6,23,24]. For the contact bi-potential $b_{c}$, given by (29), provided that $\dot{u}_{n} \geqslant 0$ and $\mathbf{r} \in K_{\mu}$, we have

$\forall \mathbf{r}^{\prime} \in K_{\mu}$

$\varrho \mu\left(r_{n}^{\prime}-r_{n}\right)\left\|-\dot{\mathbf{u}}_{t}\right\|+\left(\mathbf{r}^{\prime}-(\mathbf{r}-\varrho \dot{\mathbf{u}})\right) \cdot\left(\mathbf{r}^{\prime}-\mathbf{r}\right) \geqslant 0$,

where $\varrho$ is a solution parameter which is not user-defined. In order to ensure numerical convergence, $\varrho$ can be chosen as the maximum value of the diagonal terms of the local contact stiffness matrix. Taking account of decomposition (21), (22), the following inequality has to be satisfied:

$\mathbf{r}^{\prime} \in K_{\mu}, \quad(\mathbf{r}-\tau) \cdot\left(\mathbf{r}^{\prime}-\mathbf{r}\right) \geqslant 0$,

where the modified augmented surface traction $\tau$ is defined by

$\tau=\mathbf{r}+\varrho\left(-\dot{\mathbf{u}}_{t}-\left(\dot{u}_{n}+\mu\left\|-\dot{\mathbf{u}}_{t}\right\|\right) \mathbf{n}\right)$.

Inequality (31) means that $\mathbf{r}$ is the projection of $\tau$ onto the closed convex Coulomb's cone:

$\mathbf{r}=\operatorname{proj}\left(\tau, K_{\mu}\right)$
For the numerical solution of the implicit (33), Uzawa's algorithm can be used, which leads to an iterative process involving one predictor-corrector step:

Predictor $\tau^{i+1}=\mathbf{r}^{i}+\varrho^{i}\left(-\dot{\mathbf{u}}_{t}^{i}-\left(\dot{u}_{n}^{i}+\mu\left\|-\dot{\mathbf{u}}_{t}^{i}\right\|\right) \mathbf{n}\right)$,

Corrector $\mathbf{r}^{i+1}=\operatorname{proj}\left(\tau^{i+1}, K_{\mu}\right)$.

It is worth noting that, in this algorithm, the unilateral contact and the friction are coupled via the bi-potential. Another gist of the bi-potential method is that the corrector can be analytically found with respect to the three possible contact statuses: $\tau \subset$ $K_{\mu}$ (contact with sticking), $\tau \subset K_{\mu}^{*}$ (no contact) and $\tau \subset$ $\mathbb{R}^{3}-K_{\mu} \cup K_{\mu}^{*}$ (contact with sliding). $K_{\mu}^{*}$ is the polar cone of $K_{\mu}$. This corrector step is explicitly given as follows:

$$
\begin{array}{lll}
\text { if } \quad \mu\left\|\tau_{t}^{i+1}\right\|<-\tau_{n}^{i+1} \text { then } \mathbf{r}^{i+1}=0 & \text { ! separating } \\
\text { else if }\left\|\tau_{t}^{i+1}\right\|<\mu \tau_{n}^{i+1} \text { then } \mathbf{r}^{i+1}=\tau^{i+1} & & \text { ! sticking } \\
\text { else } & \mathbf{r}^{i+1}=\tau^{i+1}-\frac{\left(\left\|\tau_{t}^{i+1}\right\|-\mu \tau_{n}^{i+1}\right)}{\left(1+\mu^{2}\right)} & \\
& & \\
& \times\left(\frac{\tau_{t}^{i+1}}{\left\|\tau_{t}^{i+1}\right\|}+\mu \mathbf{n}\right) & \text { ! sliding. }
\end{array}
$$

\section{Stress-strain tangent operator of Ogden's materials}

In order to construct the tangent stiffness matrix for the analysis of dynamic contact by the finite element method in the next section, it is necessary to determine the stress-strain tangent operator $\mathbb{D}$ for Ogden's law. This fourth-order tensor is obtained from the derivative of $\mathbf{S}$ with respect to $\mathbf{E}$ in Eq. (16):

$\mathbb{D}=\frac{\partial \mathbf{S}}{\partial \mathbf{E}}=4 \frac{\partial^{2} W}{\partial \mathbf{C}^{2}}=4 \sum_{i=1}^{N} \frac{\mu_{i}}{\alpha_{i}} \frac{\partial^{2} W_{1}^{i}}{\partial \mathbf{C}^{2}}+4 \sum_{i=1}^{N} \frac{\mu_{i}}{\alpha_{i} \beta_{i}} \frac{\partial^{2} W_{2}^{i}}{\partial \mathbf{C}^{2}}$

with

$W_{1}^{i}=\lambda_{1}^{\alpha_{i} / 2}+\lambda_{2}^{\alpha_{i} / 2}+\lambda_{3}^{\alpha_{i} / 2}, \quad W_{2}^{i}=J^{-\alpha_{i} \beta_{i}}=I_{3}^{-\alpha_{i} \beta_{i} / 2}$.

For the sake of simplicity, we note $\alpha=\alpha_{i} / 2$ and $\beta=-\alpha_{i} \beta_{i} / 2$. Eq. (37) becomes then

$W_{1}^{i}=\lambda_{1}^{\alpha}+\lambda_{2}^{\alpha}+\lambda_{3}^{\alpha}, \quad W_{2}^{i}=I_{3}^{\beta}$.

Simo and Taylor have provided a closed form expression in the most general case of the spatial and material tangent moduli and applied it to Ogden's material [25]. We propose in this study a similar formulation by using a spectral decomposition expressed in terms of the eigenvalue-bases $\mathbf{M}_{a}(a=1,2,3)$ of C. Furthermore, we apply a limiting technique to deal with the special case of coalescent eigenvalues. To this end, we propose a coordinate-free formulation for the spectral decomposition of C. First, we introduce four isotropic functions of $\mathbf{C}$ :

$$
\begin{aligned}
& f\left(I_{1}, I_{2}, I_{3}\right)=I_{1}^{2}-3 I_{2}, \\
& g\left(I_{1}, I_{2}, I_{3}\right)=27 I_{3}^{2}+4 I_{2}^{3}-I_{1}^{2} I_{2}^{2}+4 I_{1}^{3} I_{3}-18 I_{1} I_{2} I_{3}, \\
& h\left(I_{1}, I_{2}, I_{3}\right)=2 I_{1}^{3} / 27-I_{1} I_{2} / 3+I_{3},
\end{aligned}
$$


$\omega\left(I_{1}, I_{2}, I_{3}\right)=\frac{1}{3} \cos ^{-1}\left[\frac{2 I_{1}^{3}-9 I_{1} I_{2}+27 I_{3}}{2\left(I_{1}^{2}-3 I_{2}\right)^{3 / 2}}\right]$

with $0 \leqslant \omega \leqslant \pi / 3$.

Then, four cases can be distinguished:

Case 1: $\lambda_{1}=\lambda_{2}=\lambda_{3}=\lambda$. This case occurs if and only if $f=0$. Then it follows that

$\mathbf{C}=\lambda \mathbf{I}$.

Case 2: $\lambda_{1}>\lambda_{2}=\lambda_{3}$. This case takes place if and only if $f \neq 0, g=0, h>0$.

When these conditions are verified, we have

$\lambda_{1}=\frac{1}{3} I_{1}+\frac{2}{3} \sqrt{I_{1}^{2}-3 I_{2}}$,

$\lambda_{2}=\lambda_{3}=\frac{1}{3} I_{1}-\frac{1}{3} \sqrt{I_{1}^{2}-3 I_{2}}$,

$\mathbf{C}=\lambda_{1} \mathbf{M}_{1}+\lambda_{3}\left(\mathbf{I}-\mathbf{M}_{1}\right)$,

$\mathbf{M}_{1}=\frac{1}{\sqrt{I_{1}^{2}-3 I_{2}}}\left[\mathbf{C}-\frac{1}{3}\left(I_{1}-\sqrt{I_{1}^{2}-3 I_{2}}\right) \mathbf{I}\right]$.

Case 3: $\lambda_{1}=\lambda_{2}>\lambda_{3}$. This case prevails if and only if $f \neq$ $0, g=0, h<0$.

Under these conditions, the following expressions are valid:

$\lambda_{1}=\lambda_{2}=\frac{1}{3} I_{1}+\frac{1}{3} \sqrt{I_{1}^{2}-3 I_{2}}, \quad \lambda_{3}=\frac{1}{3} I_{1}-\frac{2}{3} \sqrt{I_{1}^{2}-3 I_{2}}$,

$\mathbf{C}=\lambda_{1}\left(\mathbf{I}-\mathbf{M}_{3}\right)+\lambda_{3} \mathbf{M}_{3}$,

$\mathbf{M}_{3}=\frac{1}{\sqrt{I_{1}^{2}-3 I_{2}}}\left[\frac{1}{3}\left(I_{1}+\sqrt{I_{1}^{2}-3 I_{2}}\right) \mathbf{I}-\mathbf{C}\right]$.

Case 4: $\lambda_{1}>\lambda_{2}>\lambda_{3}$. This case is true if and only if $f \neq$ $0, g \neq 0$.

Whenever these two conditions are satisfied, we have

$\lambda_{1}=\frac{1}{3} I_{1}+\frac{2}{3} \sqrt{I_{1}^{2}-3 I_{2}} \cos \omega$,

$\lambda_{2}=\frac{1}{3} I_{1}+\frac{2}{3} \sqrt{I_{1}^{2}-3 I_{2}} \cos \left(\frac{2}{3} \pi-\omega\right)$,

$\lambda_{3}=\frac{1}{3} I_{1}+\frac{2}{3} \sqrt{I_{1}^{2}-3 I_{2}} \cos \left(\frac{2}{3} \pi+\omega\right)$,

where the angle $\omega$ is given by Eq. (42). Correspondingly,

$\mathbf{C}=\sum_{a=1}^{3} \lambda_{a} \mathbf{M}_{a}$

$\mathbf{M}_{a}=\frac{\left(\mathbf{C}-\lambda_{b} \mathbf{I}\right)\left(\mathbf{C}-\lambda_{c} \mathbf{I}\right)}{\left(\lambda_{a}-\lambda_{b}\right)\left(\lambda_{a}-\lambda_{c}\right)}$,

where $(a, b, c)$ represents a cyclic permutation of $(1,2,3)$.

It is noted that Cases 2 and 3 can be put together by substitution so as to write $\lambda_{1}=\lambda_{2}$ and $\lambda_{1} \neq \lambda_{3}$.

In order to calculate the second derivatives of $W_{1}$ with respect to $\mathbf{C}$, a limiting technique $[10,26,27]$ was used to take account for the special case of coalescent eigenvalues in which nondifferentiability occurs. After some calculations, we obtain the second derivatives of $W_{1}$ for the case of distinct or repeated eigenvalues.

Case A: triple eigenvalues: $\lambda_{1}=\lambda_{2}=\lambda_{3}=\lambda_{3}=\lambda$

$\frac{\partial^{2} W_{1}}{\partial \mathbf{C}^{2}}=\alpha(\alpha-1) \lambda^{\alpha-2} \underline{\mathbf{I}} \underline{\overline{\mathbf{I}}}$.

Case B: double eigenvalues: $\lambda_{1}=\lambda_{2}$ and $\lambda_{1} \neq \lambda_{3}$

$$
\begin{aligned}
\frac{\partial^{2} W_{1}}{\partial \mathbf{C}^{2}}= & \alpha(\alpha-1)\left[\lambda_{1}^{\alpha-2}\left(\mathbf{I}-\mathbf{M}_{3}\right) \underline{\bar{\otimes}}\left(\mathbf{I}-\mathbf{M}_{3}\right)\right. \\
& \left.+\lambda_{3}^{\alpha-2} \mathbf{M}_{3} \otimes \mathbf{M}_{3}\right]+\alpha \frac{\lambda_{1}^{\alpha-1}-\lambda_{3}^{\alpha-1}}{\lambda_{1}-\lambda_{3}}\left[\left(\mathbf{I}-\mathbf{M}_{3}\right) \underline{\bar{\otimes}} \mathbf{M}_{3}\right. \\
& \left.+\mathbf{M}_{3} \underline{\bar{\otimes}}\left(\mathbf{I}-\mathbf{M}_{3}\right)\right] .
\end{aligned}
$$

Case C: distinct eigenvalues: $\lambda_{1} \neq \lambda_{2}, \lambda_{2} \neq \lambda_{3}$ and $\lambda_{1} \neq \lambda_{3}$

$$
\begin{aligned}
\frac{\partial^{2} W_{1}}{\partial \mathbf{C}^{2}}=\alpha & \alpha(\alpha-1) \sum_{a=1}^{3} \lambda_{a}^{\alpha-2} \mathbf{M}_{a} \otimes \mathbf{M}_{a} \\
& \left.+\sum_{a=1}^{3} \frac{\lambda_{a}^{\alpha-1}-\lambda_{b}^{\alpha-1}}{\lambda_{a}-\lambda_{b}}\left(\mathbf{M}_{a} \underline{\otimes} \mathbf{M}_{b}+\mathbf{M}_{b} \underline{\otimes} \mathbf{M}_{a}\right)\right],
\end{aligned}
$$

where $b$ changes with respect to $a$ following a cyclic permutation of $(1,2,3)$.

We now deal with the potential of the form $W_{2}^{i}=I_{3}^{\beta}$ in Eq. (38). As this potential does not have problem of differentiability encountered for the potential $W_{1}$, the calculation is then much simpler. By using the chain derivative rule and some standard results (see $[28,29]$ ), we have

$\frac{\partial^{2} W_{2}}{\partial \mathbf{C}^{2}}=\beta I_{3}^{\beta}\left(\beta \mathbf{C}^{-1} \otimes \mathbf{C}^{-1}-C^{-1} \underline{\bar{\otimes}} \mathbf{C}^{-1}\right)$.

The coordinate-free symbols $\otimes$ and $\overline{\bar{Q}}$ used above are related to the corresponding index symbols in the following way

$(\mathbf{A} \otimes \mathbf{B})_{i j k l}=A_{i j} B_{k l}$,

$(\mathbf{A} \underline{\otimes} \mathbf{B})_{i j k l}=\frac{1}{2}\left(A_{i k} B_{j l}+A_{i l} B_{j k}\right)$.

\section{Finite element formulation of non-linear structures}

\subsection{Total Lagrangian formulation}

In the case of dynamic multibody contact problems involving large deformations of hyperelastic solids, the non-linear relation between strains and displacements cannot be ignored. The total Lagrangian formulation is adopted in this work to describe nonlinear behavior. It is well known that the strain tensor $\mathbf{E}$ and the stress tensor $\mathbf{S}$ are both symmetric. Thus, we note hereafter $\mathbf{E}$ and $\mathbf{S}$ in vector form as

$\mathbf{E}=\left\langle\begin{array}{llllll}E_{11} & E_{22} & E_{33} & 2 E_{12} & 2 E_{13} & 2 E_{23}\end{array}\right\rangle^{\mathrm{T}}$,

$\mathbf{S}=\left\langle\begin{array}{llllll}S_{11} & S_{22} & S_{33} & S_{12} & S_{13} & S_{23}\end{array}\right\rangle^{\mathrm{T}}$.

In the context of the finite element method and with Eqs. (4) and (6), the Green-Lagrangian strain can be formally written with 
linear and non-linear terms in terms of nodal displacements [21]:

$\mathbf{E}=\left(\mathbf{B}_{\mathrm{L}}+\frac{1}{2} \mathbf{B}_{\mathrm{NL}}(\mathbf{u})\right) \mathbf{u}$,

where $\mathbf{B}_{\mathrm{L}}$ is the matrix which relates the linear strain term to the nodal displacements, and $\mathbf{B}_{\mathrm{NL}}(\mathbf{u})$, the matrix which relates the non-linear strain term to the nodal displacements. From Eq. (61), the incremental form of the strain-displacement relationship is

$\delta \mathbf{E}=\left(\mathbf{B}_{\mathrm{L}}+\mathbf{B}_{\mathrm{NL}}(\mathbf{u})\right) \delta \mathbf{u}$.

Using the principle of virtual displacement, the virtual work $\delta U$ is given as

$$
\begin{aligned}
\delta U= & \delta \mathbf{u}^{\mathrm{T}} \mathbf{M} \ddot{\mathbf{u}}+\delta \mathbf{u}^{\mathrm{T}} \mathbf{A} \dot{\mathbf{u}}+\int_{V_{0}} \delta \mathbf{E}^{\mathrm{T}} \mathbf{S} \mathrm{d} V \\
& -\delta \mathbf{u}^{\mathrm{T}} \mathbf{F}_{\mathrm{ext}}-\delta \mathbf{u}^{\mathrm{T}} \mathbf{R}=0,
\end{aligned}
$$

where $V_{0}$ is the volume of the initial configuration, $\mathbf{F}_{\text {ext }}$ the vector of external loads, $\mathbf{R}$ the contact reaction vector, $\mathbf{M}$ the mass matrix, A the damping matrix, $\dot{\mathbf{u}}$ the velocity vector and $\ddot{\mathbf{u}}$ the acceleration vector. In view of Eqs. (36), (60) and (62), we obtain

$\delta \mathbf{S}=\mathbf{D} \delta \mathbf{E}=\mathbf{D}\left(\mathbf{B}_{\mathrm{L}}+\mathbf{B}_{\mathrm{NL}}(\mathbf{u})\right) \delta \mathbf{u}$,

where $\mathbf{D}$ denotes the constitutive tangent matrix which is deduced from the stress-strain tangent operator (Eqs. (36)-(58)) due to its major and minor symmetries: with the initial conditions at $t=0$,

$\dot{\mathbf{u}}=\dot{\mathbf{u}}_{0} \quad$ and $\quad \mathbf{u}=\mathbf{u}_{0}$.

Taking the derivative of $\mathbf{F}_{\text {int }}$ with respect to the nodal displacements $\mathbf{u}$ gives the tangent stiffness matrix as

$\mathbf{K}=\frac{\partial \mathbf{F}_{\text {int }}}{\partial \mathbf{u}}=\mathbf{K}_{e}+\mathbf{K}_{\sigma}+\mathbf{K}_{u}$

where $\mathbf{K}_{e}, \mathbf{K}_{\sigma}$ and $\mathbf{K}_{u}$ stand respectively for the elastic stiffness matrix, the geometric stiffness (or initial stress stiffness) matrix and the initial displacement stiffness matrix:

$\mathbf{K}_{e}=\int_{V_{0}} \mathbf{B}_{\mathrm{L}}^{\mathrm{T}} \mathbf{D} \mathbf{B}_{\mathrm{L}} \mathrm{d} V$

$\mathbf{K}_{\sigma}=\int_{V_{0}} \frac{\partial \mathbf{B}_{\mathrm{NL}}^{\mathrm{T}}}{\partial \mathbf{u}} \mathbf{S} \mathrm{d} V$

$\mathbf{K}_{u}=\int_{V_{0}}\left(\mathbf{B}_{\mathrm{L}}^{\mathrm{T}} \mathbf{D} \mathbf{B}_{\mathrm{NL}}+\mathbf{B}_{\mathrm{NL}}^{\mathrm{T}} \mathbf{D} \mathbf{B}_{\mathrm{L}}+\mathbf{B}_{\mathrm{NL}}^{\mathrm{T}} \mathbf{D} \mathbf{B}_{\mathrm{NL}}\right) \mathrm{d} V$.

\subsection{First-order integration algorithm}

We can now integrate Eq. (69) between consecutive time configuration $t$ and $t+\Delta t$. The most common method to do that is the Newmark method which is a second order algorithm. However, in impact problems, higher order approximation does not necessarily mean better accuracy, and may even be

$\mathbf{D}=\left[\begin{array}{cccccc}D_{1111} & D_{1122} & D_{1133} & D_{1112} & D_{1113} & D_{1123} \\ D_{1122} & D_{222} & D_{2233} & D_{2212} & D_{2213} & D_{2223} \\ D_{1133} & D_{2233} & D_{3333} & D_{3312} & D_{3313} & D_{3323} \\ D_{1112} & D_{2212} & D_{3312} & \frac{\left(D_{1212}+D_{1221}\right)}{2} & \frac{\left(D_{1213}+D_{1231}\right)}{2} & \frac{\left(D_{1223}+D_{1232}\right)}{2} \\ D_{1113} & D_{2213} & D_{3313} & \frac{\left(D_{1312}+D_{1321}\right)}{2} & \frac{\left(D_{1313}+D_{1331}\right)}{2} & \frac{\left(D_{1323}+D_{1332}\right)}{2} \\ D_{1123} & D_{2223} & D_{3323} & \frac{\left(D_{2312}+D_{2321}\right)}{2} & \frac{\left(D_{2313}+D_{2331}\right)}{2} & \frac{\left(D_{2323}+D_{2332}\right)}{2}\end{array}\right]$.

Substituting $\delta \mathbf{E}$ from Eq. (62) into Eq. (63) results in

$$
\begin{aligned}
\delta U= & \delta \mathbf{u}^{\mathrm{T}} \mathbf{M} \ddot{\mathbf{u}}+\delta \mathbf{u}^{\mathrm{T}} \mathbf{A} \dot{\mathbf{u}}+\delta \mathbf{u}^{\mathrm{T}} \int_{V_{0}}\left(\mathbf{B}_{\mathrm{L}}+\mathbf{B}_{\mathrm{NL}}(\mathbf{u})\right)^{\mathrm{T}} \mathbf{S} \mathrm{d} V \\
& -\delta \mathbf{u}^{\mathrm{T}} \mathbf{F}_{\mathrm{ext}}-\delta \mathbf{u}^{\mathrm{T}} \mathbf{R}=0 .
\end{aligned}
$$

The vector of internal forces is defined by

$\mathbf{F}_{\text {int }}=\int_{V_{0}}\left(\mathbf{B}_{\mathrm{L}}+\mathbf{B}_{\mathrm{NL}}(\mathbf{u})\right)^{\mathrm{T}} \mathbf{S} \mathrm{d} V$.

Since $\delta \mathbf{u}$ is arbitrary, a set of non-linear equations can be obtained as

$\mathbf{M u ̈}+\mathbf{A} \dot{\mathbf{u}}+\mathbf{F}_{\text {int }}-\mathbf{F}_{\text {ext }}-\mathbf{R}=0$.

It is noted that the stiffness effect is taken into account by the internal forces vector $\mathbf{F}_{\text {int }}$. Eq. (68) can be transformed into

$\mathbf{M u ̈}=\mathbf{F}+\mathbf{R}, \quad \mathbf{F}=\mathbf{F}_{\text {ext }}-\mathbf{F}_{\text {int }}-\mathbf{A} \dot{\mathbf{u}}$ superfluous. At the moment of a sudden change of contact conditions (impact, release of contact), the velocity and acceleration are not continuous, and excessive regularity constraints may lead to serious errors. For this reason, the first-order algorithm proposed by Jean [15] is used in this work. This algorithm is based on the following approximations:

$\int_{t}^{t+\Delta t} \mathbf{M} \mathrm{d} \dot{\mathbf{u}}=\mathbf{M}\left(\dot{\mathbf{u}}^{t+\Delta t}-\dot{\mathbf{u}}^{t}\right)$,

$\int_{t}^{t+\Delta t} \mathbf{F} \mathrm{d} t=\Delta t\left((1-\xi) \mathbf{F}^{t}+\xi \mathbf{F}^{t+\Delta t}\right)$,

$\int_{t}^{t+\Delta t} \mathbf{R} \mathrm{d} t=\Delta t \mathbf{R}^{t+\Delta t}$

$\mathbf{u}^{t+\Delta t}-\mathbf{u}^{t}=\Delta t\left((1-\theta) \dot{\mathbf{u}}^{t}+\theta \dot{\mathbf{u}}^{t+\Delta t}\right)$,

where $0 \leqslant \xi \leqslant 1$ and $0 \leqslant \theta \leqslant 1$. In the iterative solution procedure, all the values at time $t+\Delta t$ are replaced by the values 
of the current iteration $i+1$; for example, $\mathbf{F}^{t+\Delta t}=\mathbf{F}^{i+1}$. A standard approximation of $\mathbf{F}^{i+1}$ gives

$$
\begin{aligned}
\mathbf{F}^{i+1} & =\mathbf{F}_{\mathrm{int}}^{i}+\frac{\partial \mathbf{F}}{\partial \mathbf{u}}\left(\mathbf{u}^{i+1}-\mathbf{u}^{i}\right)+\frac{\partial \mathbf{F}}{\partial \dot{\mathbf{u}}}\left(\dot{\mathbf{u}}^{i+1}-\dot{\mathbf{u}}^{i}\right) \\
& =\mathbf{F}_{\mathrm{int}}^{i}-\mathbf{K}^{i} \Delta \mathbf{u}-\mathbf{A}^{i} \Delta \dot{\mathbf{u}} .
\end{aligned}
$$

Finally, we obtain the recursive form of Eq. (69) in terms of displacements:

$\overline{\mathbf{K}}^{i} \Delta \mathbf{u}=\overline{\mathbf{F}}^{i}+\overline{\mathbf{F}}_{\text {acc }}^{i}+\mathbf{R}^{i+1}$,

$\mathbf{u}^{i+1}=\mathbf{u}^{i}+\Delta \mathbf{u}$,

where the so-called effective terms are given by

$\overline{\mathbf{K}}^{i}=\xi \mathbf{K}^{i}+\frac{\xi}{\theta \Delta t} \mathbf{A}^{i}+\frac{1}{\theta \Delta t^{2}} \mathbf{M}^{i}$,

$\overline{\mathbf{F}}_{\text {acc }}^{i}=-\frac{1}{\theta \Delta t^{2}} \mathbf{M}^{i}\left\{\mathbf{u}^{i}-\mathbf{u}^{t}-\Delta t \dot{\mathbf{u}}^{t}\right\}$,

$\overline{\mathbf{F}}^{i}=(1-\xi)\left(\mathbf{F}_{\mathrm{int}}^{t}+\mathbf{F}_{\mathrm{ext}}^{t}\right)+\xi\left(\mathbf{F}_{\mathrm{int}}^{i}+\mathbf{F}_{\mathrm{ext}}^{t+\Delta t}\right)$.

At the end of each time step, the velocity is updated by

$\dot{\mathbf{u}}^{t+\Delta t}=\left(1-\frac{1}{\theta}\right) \dot{\mathbf{u}}^{t}+\frac{1}{\theta \Delta t}\left(\mathbf{u}^{t+\Delta t}-\mathbf{u}^{t}\right)$.

Eq. (80) is strongly non-linear, because large rotations and large deformations are involved. Besides, in multibody contact/impact problems, unilateral contact and friction, characterized by inequalities, are non-smooth phenomena. To solve this equation instead of considering all non-linearities at the same time, Feng [7] has proposed a strategy which consists in separating the non-linearities so as to overcome the complexity of calculation and to improve the numerical stability. As $\Delta \mathbf{u}$ and $\mathbf{R}$ are both unknown, Eq. (80) cannot be directly solved. First, the vector $\mathbf{R}$ is determined by the bi-potential method in a reduced system, which only concerns contact nodes. The reader can refer to $[4,5,7,9]$ for more details on the bi-potential method. Then, the vector $\Delta \mathbf{u}$ can be computed in the whole structure, using contact reactions as external loading. It is important to note that, as opposed to the penalty method or Lagrange multiplier method, the bi-potential method neither changes the global stiffness matrix nor increases the degrees of freedom. This interesting property makes it easy to implement contact and friction problems in an existing general-purpose finite element code by this method. In addition, the solution procedure is more stable because of the separation of non-linearities and improved numerical algorithms for calculation of contact reactions.

\subsection{Energy computation}

After determining the displacement and the velocity fields, we can calculate different energies. From Eq. (11), we calculate the strain energy density $W$ on an integration point (Gauss point). The total elastic strain energy of the contact bodies (discretized by $n_{\mathrm{el}}$ finite elements) is then written by

$E_{e}=\sum_{e=1}^{n_{\mathrm{el}}} \int_{V_{e}} W \mathrm{~d} V$.
The total kinetic energy can be calculated at the global level by

$E_{k}=\frac{1}{2} \dot{\mathbf{u}}^{\mathrm{T}} \mathbf{M} \dot{\mathbf{u}}$.

Finally, the total energy of the system of solids is

$E_{t}=E_{e}+E_{k}$

The case of interest for the analysis presented below corresponds to the homogeneous Neumann problem, characterized by no imposed boundary displacements and no external loading. In addition, if frictionless contact is considered, the total energy should be conserved. The given example shows that this fundamental energy conservation property is preserved.

\section{A numerical example}

The algorithms presented above have been implemented and tested in the finite element code FER/Impact [30]. To illustrate the capacity of the algorithm described above to simulating contact/impact problems, we consider here one example where no damping exists except for Coulomb friction between contact surfaces, i.e., $\mathbf{A}=0$ in Eq. (81).

This problem concerns the impact of a cylinder made of rubbers upon two oblique rigid symmetric surfaces forming a funnel, inspired of the work by Wriggers et al. [31]. The characteristics of this example are: Ogden's model parameters $N=1, \mu_{1}=-3 \mathrm{MPa}, \alpha_{1}=-2$ and $\beta_{1}=0.5$, initial mass density $\rho_{0}=700 \mathrm{~kg} / \mathrm{m}^{3}$, initial velocity $v_{y}=-30 \mathrm{~m} / \mathrm{s}$. The radius of the cylinder is: $R=0.01 \mathrm{~m}$. The total simulation time is $3 \times 10^{-3} \mathrm{~s}$ and the solution parameters are: $\Delta t=10^{-5} \mathrm{~s}, \xi=\theta=0.5$. The cylinder is modeled by 209 nodes and 192 linear quadrilateral plane strain elements without considering the symmetry (Fig. 2). The initial position of the cylinder is given by its center point $O(0.0,0.03)$. The right part of the rigid block is defined by $A(0.005,0.0), B(0.015,0.0), C(0.015,0.035)$ and $D(0.012,0.035)$.

Three cases A, B and C are considered (Table 1). It is noted that these analyses were performed on a PC (Pentium $\frac{4}{2.8} \mathrm{GHz}$ ). Fig. 3 shows the von Mises stress of $\boldsymbol{\sigma}$ in the cylinder at the moment when the cylinder reaches its lowest position and the kinetic energy $E_{k}$ equals zero (see Figs. 6-8). The CPU time to

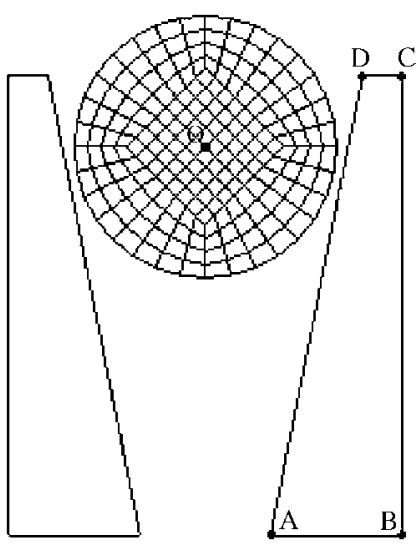

Fig. 2. Deformable-rigid impact. 
Table 1

Influence of friction coefficients

\begin{tabular}{llll}
\hline Case & At time $(\mathrm{ms})$ & $\sigma_{\max }(\mathrm{MPa})$ & CPU time $(\mathrm{s})$ \\
\hline A: $\mu=0.0$ & 0.87 & 8.192 & 62 \\
B: $\mu=0.2$ & 0.70 & 4.523 & 77 \\
C: $\mu=0.4$ & 0.61 & 4.396 & 83 \\
\hline
\end{tabular}

achieve the solution and the maximum value of the von Mises stress (relative to the Cauchy stress) are also given in Table 1. We observe apparent differences concerning the values and the localization of the maximum stress. In Case A, the cylinder goes down lower so as to be more deformed. Therefore, the stress value is higher. When the friction increases, the cylinder does not go down as low as before, braked by the friction forces. The localization of the maximum stress moves to the contact surfaces because friction forces are added onto the surfaces in contact, as shown in Fig. 3. The deformed configurations at time $t=2 \mathrm{~ms}$ are shown in Fig. 4. The displacement of the center point $O$ versus time is plotted in Fig. 5. For the three cases, Figs. 6-8 show the plots of the kinetic energy $E_{k}$, the elastic strain energy $E_{e}$ and the total energy $E_{t}$. For Case A, the cylinder rebounds with the same velocity as the initial velocity, as there is no energy loss in the system (see Fig. 6). For Case $\mathrm{B}$, the cylinder rebounds as well but with smaller velocity than the initial velocity, as there is energy loss in the system (see Fig. 7). By taking into account more important friction (Case C), after a jump up, the cylinder sticks to the contact surfaces and the kinetic energy $E_{k}$ tends to zero and thus the cylinder is locked (see Fig. 8). Furthermore, the elastic deformation is smaller, as compared to the frictionless case A.

We observe that the total energy is quite well conserved in the case of frictionless contact (Fig. 6). However, in the case of frictional contact, the total energy decreases (Figs. 7 and 8). So the total energy is dissipated by frictional effects as expected. It is worth noting that the dissipated energy is quantitatively calculated. It is also interesting to examine another question: is the dissipated energy proportional to the friction coefficient? The answer is negative according to numerical results. This can be seen by Figs. 7 and 8 in which we observe almost the same dissipated energy with two different friction coefficients. In fact, when the friction coefficient increases, the friction forces increase but the tangential slips decrease (Fig. 3).

In this study, another interesting result is obtained by comparing cases B and C. In Case B (Fig. 7), the kinetic energy tends to a constant value and the elastic strain energy equals zero after the impact. On the contrary, in Case C (Fig. 8), the kinetic energy tends to zero and the elastic strain energy is not released. As expected, the cylinder is locked inside the funnel. For both cases, the total energy remains almost the same. Thus, friction is seen to play a key role in contact/impact problems.

In Case $\mathrm{C}$, we have changed the parameter $\beta(0.01,0.1,0.5$, 5.0) so as to check its influence on the model. Fig. 9 shows the evolution of the total energy. Fig. 10 depicts the evolution of the von Mises stress at the center of the cylinder (point $O$ in Fig. 2). These results indicate the tendency that the incompressibility of the Ogden's materials increases when $\beta$ decreases.

In order to test the robustness of the proposed algorithm, we reconsider the frictionless impact case (Case A) and apply additionally an initial rotation of $\omega=1000 \mathrm{rad} / \mathrm{s}$ to the cylinder (namely Case D). Fig. 11 shows the initial and deformed configurations at time $t=0.6 \mathrm{~ms}$. The rotation effects can be clearly shown by means of the evolution of three specific points $\mathrm{H}, O$ and $\mathrm{G}$. The plots of the kinetic energy $E_{k}$, the elastic strain energy $E_{e}$ and the total energy $E_{t}$ are given in Fig. 12. By comparing Figs. 6 and 12, we observe that the evolution of the elastic strain energy remains the same for Case A (without initial rotation) and Case $\mathrm{D}$ (with initial rotation). The kinetic energy is just shifted higher in Case D as compared to Case A due to the contribution of the initial rotation. It is also

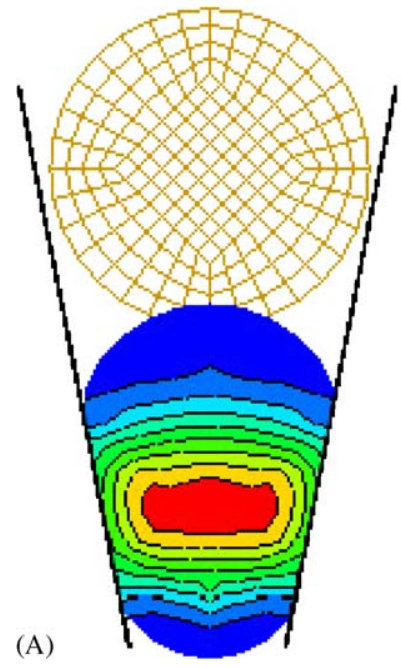

(B)

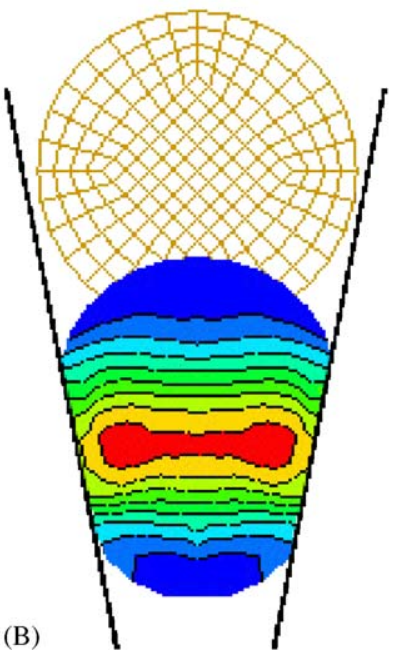

(C)

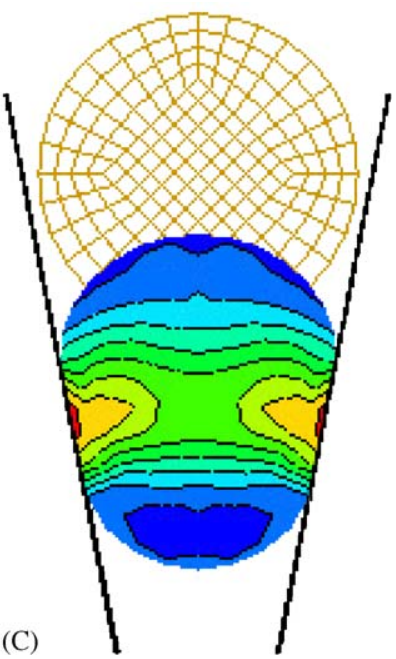

Fig. 3. Isovalues of von Mises stress. 




Fig. 4. Deformed configurations at time $t=2 \mathrm{~ms}$.

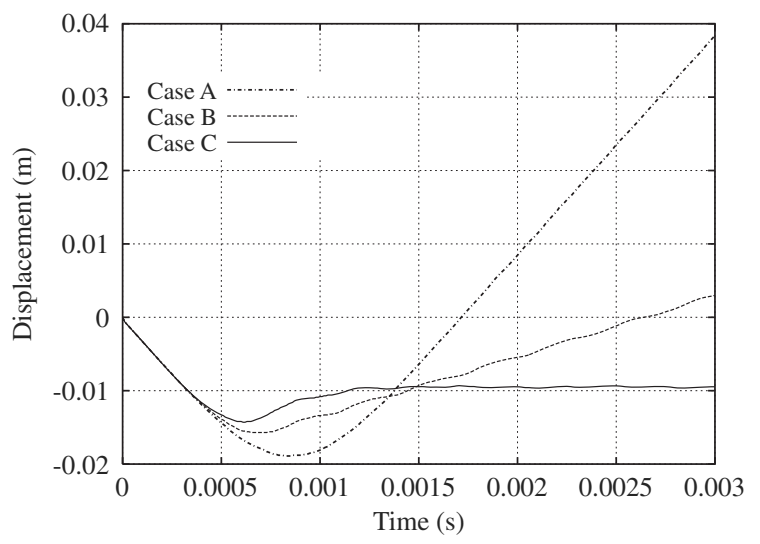

Fig. 5. Displacement of point $O$.

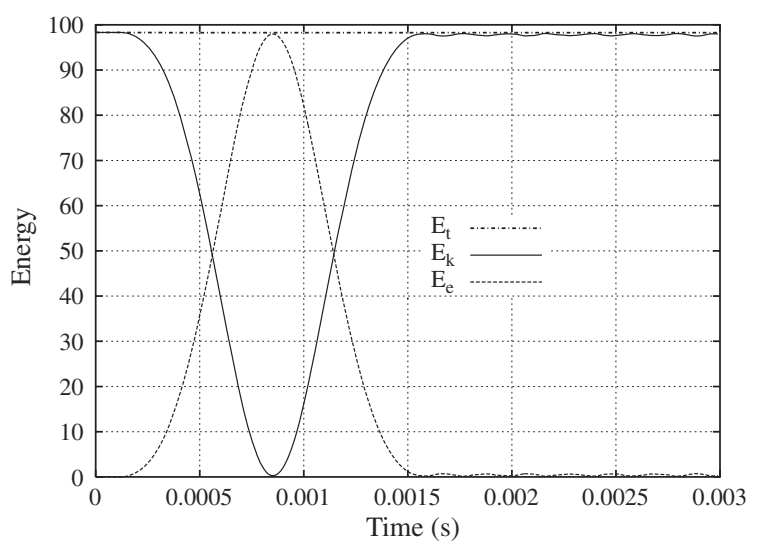

Fig. 6. Energy evolution $(\mu=0.0)$.

observed that, in both cases, the total energy is well conserved. These results indicate that the initial rotation does not really affect the impact behavior of solids. Of course, this is true only if frictionless contact is considered.

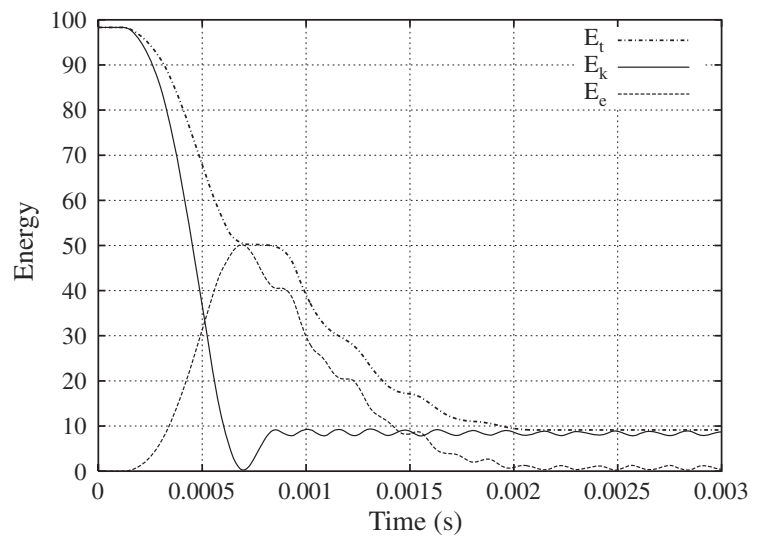

Fig. 7. Energy evolution $(\mu=0.2)$.



Fig. 8. Energy evolution $(\mu=0.4)$.

\section{Concluding remarks}

In this work, Ogden's hyperelastic law has been theoretically investigated and numerically implemented in the context 


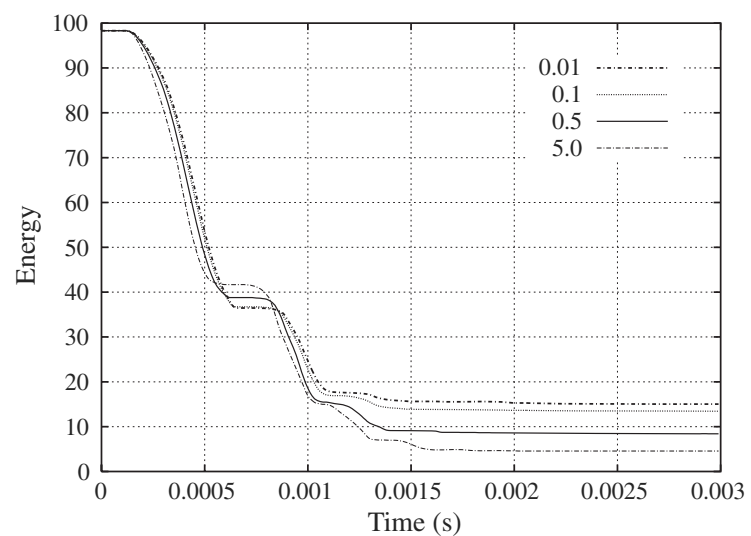

Fig. 9. Total energy.



Fig. 10. von Mises stress.



Fig. 11. Initial and deformed configurations at time $t=0.6 \mathrm{~ms}$ (with initial rotation).

of impact loading with friction. The coupling of the bi-potential method and the first-order numerical integration scheme has given rise to an algorithm which allows to simulate the behavior of Ogden's materials under impact loading in a numerically stable and energy conserving way. The cases where the



Fig. 12. Energy evolution $(\mu=0.0)$ (with initial rotation).

eigenvalues of the right Cauchy-Green strain tensors $\mathbf{C}$ are not single have been carefully treated for the finite element implementation. The numerical experiments performed indicate that:

- the total energy is well conserved in the case of frictionless impact with or without initial rotation;

- the physical energy dissipation due to friction is quantitatively determined;

- the locking of the impacting body by friction effects is numerically captured.

The algorithm presented in this paper can be extended to threedimensional dynamic contact problems including more complex frictional models such as orthotropic friction laws with non-associated flow rules [32]. This work is being undertaken.

\section{References}

[1] N. Kikuchi, J.T. Oden, Contact Problems in Elasticity: A Study of Variational Inequalities and Finite Elements, SIAM, Philadelphia, 1988.

[2] Z.H. Zhong, Finite Element Procedures in Contact-Impact Problems, Oxford University Press, Oxford, 1993.

[3] P. Wriggers, Computational Contact Mechanics, Wiley, New York, 2002.

[4] G. de Saxcé, Z.-Q. Feng, New inequality and functional for contact with friction: the implicit standard material approach, Mech. Struct. Mach. 19 (1991) 301-325.

[5] G. de Saxcé, Z.-Q. Feng, The bi-potential method: a constructive approach to design the complete contact law with friction and improved numerical algorithms, Math. Comput. Model. 28 (4-8) (1998) 225-245 (Special issue: Recent Advances in Contact Mechanics).

[6] A. Klarbring, Mathematical programming and augmented Lagrangian methods for frictional contact problems, in: A. Curnier (Ed.), Contact Mechanics International Symposium 1992.

[7] Z.-Q. Feng, 2D or 3D frictional contact algorithms and applications in a large deformation context, Commun. Numer. Methods Eng. 11 (1995) 409-416.

[8] Z.-Q. Feng, Some test examples of 2D and 3D contact problems involving coulomb friction and large slip, Math. Comput. Model. 28 (4-8) (1998) 469-477 (Special issue: Recent Advances in Contact Mechanics).

[9] Z.-Q. Feng, F. Peyraut, N. Labed, Solution of large deformation contact problems with friction between Blatz-Ko hyperelastic bodies, Int. J. Eng. Sci. 41 (2003) 2213-2225.

[10] R.W. Ogden, Non-Linear Elastic Deformations, Ellis Horwood, Chichester, UK, 1984.

[11] C. Kane, E.A. Repetto, M. Ortiz, J.E. Marsden, Finite element analysis of nonsmooth contact, Comput. Meth. Appl. Mech. Eng. 180 (1999) $1-26$. 
[12] L. Demkowicz, A. Bajer, Conservative discretization of contact/impact problems for nearly rigid bodies, Comput. Meth. Appl. Mech. Eng. 190 (2001) 1903-1924.

[13] T.A. Laursen, G.R. Love, Improved implicit integrators for transient impact problems geometric admissibility within the conserving framework, Int. J. Numer. Meth. Eng. 53 (2002) 245-274.

[14] G.R. Love, T.A. Laursen, Improved implicit integrators for transient impact problems: dynamic frictional dissipation within an admissible conserving framework, Comput. Meth. Appl. Mech. Eng. 192 (2003) 2223-2248.

[15] M. Jean, The non-smooth contact dynamics method, Comput. Meth. Appl. Mech. Eng. 177 (1999) 235-257.

[16] Z.-Q. Feng, P. Joli, J.-M. Cros, B. Magnain, The bi-potential method applied to the modeling of dynamic problems with friction, Comput. Mech. 36 (2005) 375-383.

[17] P.J. Blatz, W.L. Ko, Application of finite elastic theory to the deformation of rubbery materials, Trans. Soc. Rheol. 6 (1962) 223-251.

[18] P.G. Ciarlet, Elasticité Tridimensionnelle, Masson, Collection RMA 1985.

[19] E.M. Arruda, M.C. Boyce, A three dimensional constitutive model for the large deformation stretch behavior of rubber elastic materials, J. Mech. Phys. Solids 41 (1993) 389-412.

[20] A.N. Gent, A new constitutive relation for rubber, Rubber Chem. Technol. 69 (1996) 59-61.

[21] J.C. Simo, T.J.R. Hughes, Computational Inelasticity, Springer, New York, 1998.

[22] C.O. Horgan, G. Saccomandi, Finite thermoelasticity with limiting chain extensibility, J. Mech. Phys. Solids 51 (2003) 1127-1146.
[23] P. Alart, A. Curnier, A mixed formulation for frictional contact problems prone to Newton like solution methods, Comput. Meth. Appl. Mech. Eng. 92 (1991) 353-375.

[24] J.C. Simo, T.A. Laursen, An augmented Lagrangian treatment of contact problems involving friction, Comput. Struct. 42 (1992) 97-116.

[25] J.C. Simo, R.L. Taylor, Quasi-incompressible finite elasticity in principal stretches, continuum basis and numerical algorithms, Comput. Meth. Appl. Mech. Eng. 85 (1991) 273-310.

[26] A.F. Saleeb, T.Y.P. Chang, S.M. Arnold, On the development of explicit robust schemes for implementation of a class of hyperelastic models in large-strain analysis of rubbers, Int. J. Numer. Meth. Eng. 33 (1992) 1237-1249.

[27] Y. Basar, M. Itskov, Finite element formulation of the Ogden material model with application to rubber-like shells, Int. J. Numer. Meth. Eng. 42 (1998) 1279-1305.

[28] C. Miehe, Computation of isotropic tensor functions, Commun. Numer. Meth. Eng. 9 (1993) 889-896.

[29] J. Schroder, P. Neff, Invariant formulation of hyperelastic transverse isotropy based on polyconvex free energy functions, Int. J. Solids Struct. 40 (2003) 401-445

[30] Z.-Q. Feng, 〈http://gmfe16.cemif.univ-evry.fr:8080/ feng/FerImpact. html $\rangle$.

[31] P. Wriggers, T. Vu Van, E. Stein, Finite element formulation of large deformation impact contact problems with friction, Comput. Struct. 37 (1990) 319-331.

[32] M. Hjiaj, Z.-Q. Feng, G. de Saxcé, Z. Mróz, There-dimensional finite element computations for frictional contact problems with non-associated sliding rule, Int. J. Numer. Meth. Eng. 60 (2004) 2045-2076. 